\title{
Dynamic Infrared Imaging for Skin Cancer Screening
}

\author{
Sebastián E. Godoy ${ }^{\mathrm{a}, \mathrm{b}}$, David A. Ramirez ${ }^{\mathrm{c}}$, Stephen A. Myers ${ }^{\mathrm{c}}$, Greg von Winckel ${ }^{\mathrm{c}}$, \\ Sanchita Krishna ${ }^{\mathrm{c}}$, Marianne Berwick ${ }^{\mathrm{d}, \mathrm{e}}$, R. Steven Padilla ${ }^{\mathrm{d}, \mathrm{e}}$, Pradeep Sen ${ }^{\mathrm{f}}$ and \\ Sanjay Krishna, a,c,1 \\ ${ }^{a}$ Electrical and Computer Engineering (ECE), University of New Mexico, 1 University of New \\ Mexico, MSC01 1100, Albuquerque, NM 87131-0001, USA \\ ${ }^{b}$ Center for High Technology Materials (CHTM), University of New Mexico, 1313 Goddard \\ Street SE, MSC04 2710, Albuquerque, NM 87106-4343, USA \\ ${ }^{c}$ Skinfrared, LLC, 801 University Blvd. SE, Suite 100, Albuquerque, NM, 87106, USA \\ ${ }^{d}$ UNM Cancer Center, 1201 Camino de Salud NE, 1 University of New Mexico, Albuquerque, \\ NM 87106 \\ ${ }^{e}$ UNM Department of Dermatology, 1021 Medical Arts NE Albuquerque, NM 87131 \\ ${ }^{f}$ Department of Electrical and Computer Engineering, University of California, Santa Barbara, \\ Santa Barbara, CA 93106
}

Abstract: Dynamic thermal imaging (DTI) with infrared cameras is a non-invasive technique with the ability to detect the most common types of skin cancer. We discuss and propose a standardized analysis method for DTI of actual patient data, which achieves high levels of sensitivity and specificity by judiciously selecting pixels with the same initial temperature. This process compensates the intrinsic limitations of the cooling unit and is the key enabling tool in the DTI data analysis. We have extensively tested the methodology on human subjects using thermal infrared image sequences from a pilot study conducted jointly with the University of New Mexico Dermatology Clinic in Albuquerque, New Mexico (ClinicalTrials ID number NCT02154451). All individuals were adult subjects who were scheduled for biopsy or adult volunteers with clinically diagnosed benign condition. The sample size was 102 subjects for the present study. Statistically significant results were obtained that allowed us to distinguish between

\footnotetext{
${ }^{1}$ Corresponding author:

Center for High Technology Materials (CHTM), University of New Mexico,

1313 Goddard Street SE, MSC04 2710, Albuquerque, NM 87106-4343, USA

Phone: +1 (505) 272 7800;

Fax: +1 (505) 272 7801;

Email: skrishna@chtm.unm.edu
} 
benign and malignant skin conditions. The sensitivity and specificity was $95 \%$ (with a $95 \%$ confidence interval of $[87.8 \% 100.0 \%]$ ) and $83 \%$ (with a 95\% confidence interval of [73.4\% 92.5\%]), respectively, and with an area under the curve of $95 \%$. Our results lead us to conclude that the DTI approach in conjunction with the judicious selection of pixels has the potential to provide a fast, accurate, non-contact, and non-invasive way to screen for common types of skin cancer. As such, it has the potential to significantly reduce the number of biopsies performed on suspicious lesions.

PACS-2010 Codes: 42.30.Sy, 42.30.Tz, 42.30.Va, 44.90.+c

Keywords: skin cancer, infrared imaging, dynamic thermal imaging, skin cancer screening, non-invasive screening,

\section{Introduction}

Skin cancer is the most common form of cancer in the United States with over 3.5 million cases of skin cancer reported annually [1]. There is a higher incidence of skin cancer than the combined occurrence of breast, prostate, lung and colon cancers [2]. Melanoma, which accounts for an estimated 4\% of skin cancer cases, is responsible for approximately $75 \%$ of all deaths from skin cancer. The total deaths in the United States due to melanomas and other types of skin cancer are estimated to be more than 12,000 for $2014[1]$.

Currently, the detection of melanoma relies on a subjective ABCDE (Asymmetry, Border, Color, Diameter and Evolution) test performed visually by dermatologists, general practitioners (GP) or primary care physicians (PCP) [3]. However, the ABCDE test provides a qualitative guideline and it requires a trained specialist to actually distinguish malignant lesions from benign nevi. Moreover, the $\mathrm{ABCDE}$ approach has a relatively low specificity $(56 \%-65 \%)$ and moderate sensitivity (47\% to $89 \%)$ [3-5]. Since a false negative could lead to metastatic cancer and death, excisional biopsies are routinely performed even on lesions that are non-cancerous. For example, the number of biopsies undertaken in nine geographical areas of the US between 1986 and 2001 is close to 60 for every melanoma detected [6]. 
Since biopsies are intrusive and can be painful, different non-invasive techniques are being researched in order to minimize the number of excess biopsies performed $[7,8]$. Some of these techniques include multispectral (MS) imaging [9-11], digital dermatoscopy and videodermatoscopy (sequential digital dermatoscopy) [12, 13], reflectance-mode confocal microscopy [14], ultrasound [15, 16], laser Doppler perfusion imaging [17], and optical coherence tomography (OCT) [18, 19], to name a few. Currently, there are two FDA-approved non-invasive imaging devices MelaFind ${ }^{\mathrm{TM}}$ and Vivosight Multi-Beam System $^{\mathrm{TM}}$, which are based on MS imaging and OCT, respectively. On one hand, the MelaFind technology only works with pigmented melanomas and the specificity is as low as 9.5\% [20]. On the other hand, Vivosight presents sensitivity between $79-94 \%$ and specificity between $85-96 \%$ for nonmelanoma skin cancer lesions [21] but the suspicious lesion must be probed several times, which makes the acquisition time prohibitively high.

In order to address this problem, different groups have investigated the utilization of dynamic thermal imaging (DTI) for skin cancer screening and diagnosis [22]. For example, Anbar [23] described how changes in human skin temperature convey valuable physiological and pathophysiological information. Buzug et al. [24] and Centigül and Herman [25] studied the diagnosis of BCC and MM lesions, respectively. Both used similar techniques, requiring the cooling of the lesion to observe the warm-up pattern. Their work established that the difference in the thermal recovery may contain useful information that has the potential to non-invasively differentiate malignant lesions from benign. Nevertheless, there is not a standardized protocol to analyze the subject data such that malignant lesions are identified with high sensitivity while at the same time ensuring that benign lesions are identified with high specificity.

In this paper, we address this problem by presenting a standardized analysis protocol of DTI that judiciously selects pixels with the same initial temperature in order to compensate deficiencies in the cooling process, which, at the same time, is the key process that enables the classification of the lesion condition with an specificity $>80 \%$ for a $95 \%$ of sensitivity. We evaluate its effectiveness by presenting results from a cross-sectional sample of 102 subjects. 


\section{Material and methods}

\subsection{Data acquisition equipment}

The data acquisition equipment consists of four components. First, a cooling unit that is used to impart a temperature stimulus to the lesion and the surrounding skin tissue; the cooling unit was a Ranque-Hilsch vortex tube that generates an oil-free, moisture-free, ultra-quiet air flow. Second, an infrared marker, which is used for correction of involuntary movement of the subject (i.e., image registration); the marker is a square piece of plastic with a square opening in the middle. Third, the imaging portion of the system consists of a commercial visible camera to capture a reference image before the acquisition commences and a longwave infrared (LWIR) camera to capture the thermal recovery of the skin after the cool air is applied. The LWIR camera uses a 320x256 focal-plane array (FPA) of quantum-well infrared photodetectors (QWIP) operating at $60 \mathrm{~K}$. The noise equivalent temperature difference (NEDT) of the FPA is $20 \mathrm{mK}$ and the QWIP camera is fitted with a $50 \mathrm{~mm}, \mathrm{f} / 2 \mathrm{LWIR}$ lens, yielding an approximate spatial resolution of 300 microns per pixel. The QWIP camera was chosen for our pilot study because it has higher array uniformity, lower NETD and high spatial resolution as compared with other IR camera technologies $[22,28]$. Fourth, a custom computer program developed and coded by the authors, which performs image registration and undertakes the analysis of the subject data.

\subsection{Imaging procedure}

After informed consent, each subject was escorted to a designated room in the UNM Dermatology Clinic to perform the imaging procedure. The temperature of the room was controlled to be between $20{ }^{\circ} \mathrm{C}$ to 22 ${ }^{\circ} \mathrm{C}$ to make sure that all the patients were at the same temperature before applying the cooling stimulus to the area of interest. At the beginning of the procedure, a square registration marker was placed around the lesion with the lesion centered in the opening, as shown in Fig. 1. A visible image of the lesion was then taken with the digital camera for reference purposes. After collection of the visible image, a 15 second infrared image sequence of the marked area was collected to serve as a baseline. Later, the subject's skin within the marker opening was cooled for 30 seconds using the cooling unit. After cooling, the exposed 
area was allowed to warm up naturally to ambient temperature. During the cooling and warm-up phases, thermal images of the skin were captured for a total of 2 minutes at a rate of 60 frames per second. All the thermal images were recorded using an uncompressed 14-bit format. The total time required to complete the imaging procedure was less than five minutes. If the subject was scheduled for a biopsy, the biopsy was performed following the data collection by the attending dermatologist and sent to pathology for diagnosis. The biopsy results were delivered to us within the next two weeks following the imaging procedure.

\subsection{Data processing}

\subsubsection{Data registration}

Since involuntary movements cannot be avoided, image registration must be performed on the sequence of IR images. Moreover, to correctly reference the lesion location (pigmented area) in the IR sequence, the visible picture must also be spatially aligned with respect to the IR sequence; therefore, the visible image is considered as an additional frame for the registration.

The registration is conducted as follows. First, we use the Harris corner detector algorithm [26] to automatically detect the four corners of the plastic marker in the entire sequence of frames (visible + IR sequence). Second, we estimate an affine transformation matrix that maps the movement between consecutive frames (one matrix is estimated for each pair of frames). Third, we utilize the inverse of each transformation to align the entire sequence of images with respect to the first IR frame [27].

After registration, both the visible image and the entire infrared sequence are spatially aligned, generating a three-dimensional (3D) array of real numbers, that we denote by $\mathbf{u} \in \mathbb{R}^{I \times J \times K}$, where I and $\mathrm{J}$ represent the number of horizontal and vertical pixels, respectively, in the spatial domain, and $\mathrm{K}$ represents the total number of frames (including the aligned visible image). We denote any element of the cube $\boldsymbol{u}$ as $u(i, j, k)$, where $1 \leq i \leq \mathrm{I}, 1 \leq j \leq \mathrm{J}$ and $1 \leq k \leq \mathrm{K}$. The first frame in the cube (i.e., $k=1$ ) corresponds to the visible image, whereas the remaining frames correspond to the IR sequence. The value of $u(i, j, k), k \geq 2$ is referred 
to as the temperature of the $k$ th frame at the spatial location $(i, j)$. For a fixed spatial location $(i, j)$, the $(K$ 1)-dimensional vector $\boldsymbol{u}(i, j)=[u(i, j, 2) \ldots u(i, j, K)]$ is termed as the $(i, j)$ thermal recovery data.

\subsubsection{Lesion selection}

Given that the purpose of this paper is to compare the thermal recovery with respect to the surrounding skin (which is assumed to be healthy), we require knowing the outer boundary of the suspicious lesion, i.e., the pigmented area. For the purpose of this study we perform the lesion selection by displaying $u(i, j, 1)$ (i.e., the spatially aligned visible image) to the user in order to ask him/her to draw the boundary of the suspicious lesion using Matlab's imellipse function.

For this discussion, let us refer to Fig. 1. Define $U$ as the set of all the spatial locations within the opening of the marker, i.e., $U=\left\{(i, j): i_{\min } \leq i \leq i_{\max }, j_{\min } \leq j \leq j_{\max }\right\}$, where $i_{\min ,} i_{\max }, j_{\min ,} j_{\max }$ defines the biggest quadrilateral that can be drawn within the opening of the marker (red rectangle in Fig. 1); for this example the point $\left(i_{\min ,}, j_{\min }\right)$ is the upper-left corner of the quadrilateral, the point $\left(i_{\min }, j_{\max }\right)$ defines the upper-right corner, and so on. Let $L$ be the set of all spatial locations that define the suspicious lesion (selected by the user, as shown by the blue ellipse in Fig. 1). The set $N=L^{c} \cap U$ represents all of the spatial locations outside the lesion but within the quadrilateral of interest. The collections $U, L$ and $N$ will be utilized in the following section right after the non-uniform distribution of the initial temperature is addressed by a pixel wise modeling of the thermal recovery.

\subsection{Data analysis}

\subsubsection{Non-uniform cooling compensation}

If two pieces of material with the same thermal parameters (e.g., thermal diffusivity, specific heat, etc.) are cooled to two different temperatures they will have different recovery curves. This can be observed by evaluating the heat equation [29]. Therefore, to correctly compare the recovery of $L$ and $N$ sets, a uniform initial temperature must be ensured. However, due to the heterogeneous nature of skin tissue, this is 
difficult to implement via hardware. Thus, this issue is addressed via software by judiciously selecting only those pixels with the same initial temperature, which is key contribution of the presented work.

Ideally, when no noise is present, one should simply analyze the first frame in the IR sequence to determine which pixels can be selected under a certain pre-specified rule in order to have only pixels with the same temperature. Nonetheless, in the presence of noise, the first frame does not necessarily contain the real initial temperature. Thus, we modeled the thermal recovery of each pixel as a dual exponential function in time whose parameters are obtained by a non-linear least-square fitting of the thermal recovery data. Fig. 2 shows two example pixels for one patient and its corresponding model. Now for any collection of locations $U$, we denote the modeled thermal recovery of all these locations by $f_{U}(t)$.

Therefore, the initial temperature in the entire rectangle of interest, $f_{U}(0)$, will be given by the sum of the model coefficients, i.e., if the model is denoted by $f(t)=\alpha+\beta_{1} e^{\lambda}{ }_{1}^{t}+\beta_{2} e^{\lambda}{ }_{2}{ }^{t}$, then $f_{U}(0)=\left\{\left(\alpha_{i, j}+\beta_{1_{i, j}}+\beta_{2_{i, j}}\right):(i, j) \in U\right\}$. In the same way, we define the initial temperature of the pixel inside and outside the lesion, $f_{L}(0)$ and $f_{N}(0)$, respectively. To select the pixels with the same initial temperature, we define the reference temperature as the mean initial temperature across the pixels within the lesion, i.e., $T_{r e f}=\mathrm{E}\left[f_{L}(0)\right]$, where $\mathrm{E}$ is the mathematical expectation computed over all spatial locations in the set $L$. Next, search for all pixels in $U$ that are close to $T_{r e f}$, within a certain tolerance. By trial-and-error we determine that a range of $\pm 2 \%$ of $T_{\text {ref }}$ achieved a consistent selection of pixels among patients. As such, we define the collection of locations, $S=\left\{(i, j):(1-p) T_{\text {ref }}<f_{i, j}(0)<(1+p) T_{\text {ref }},(i, j) \in U\right\}$, where $p=0.02$ in our case. Now, we can define the collections $L^{*}=L \cap S$ and $N^{*}=N \cap S$, which contain locations inside and outside the lesion, respectively, and also have the same initial temperature (within the $p \%$ tolerance). If for some patient the set $N^{*}$ is null, the value of $p$ must be increased. 


\subsubsection{Quantification of lesion malignancy}

Let us denote by $\boldsymbol{u}_{L^{*}}(t)$ and $\boldsymbol{u}_{N^{*}}(t)$ the collections of all the thermal recoveries from the registered data, $\boldsymbol{u}$, such that its spatial locations are in the sets $L^{*}$ and $N^{*}$, respectively. More precisely, $\boldsymbol{u}_{L^{*}}(t)=\{\mathrm{u}(i, j, k):(i, j)$ $\left.\left.\in L^{*}, 2 \leq \mathrm{k} \leq \mathrm{K}\right\}\right)$ and $\left.\boldsymbol{u}_{N^{*}}(t)=\left\{\mathrm{u}(i, j, k):(i, j) \in N^{*}, 2 \leq \mathrm{k} \leq \mathrm{K}\right\}\right)$.

As it was proposed and quantified before [24, 25], the malignant tissue when compared with benign tissue should present different thermal parameters such as thermal diffusion, tissue specific heat, metabolic heat generation, etc. Now, if there is any difference in the thermal recovery of the pixels in $L^{*}$ with respect to the recovery of the pixels in $N^{*}$, then it is primarily due to the difference in the thermal parameters of each collection, owing that all the pixels whose locations are in $S$ have the same initial temperature. Therefore, the sub-selection of pixels ensures that, if there is any difference in the thermal recoveries in $\boldsymbol{u}_{L^{*}}(t)$ and $\boldsymbol{u}_{N^{*}}(t)$, then they are solely related to the malignancy of the suspicious lesion.

We hypothesized then that the suspicious lesion should be declared as malignant if its average thermal recovery computed across the locations $L^{*}$, i.e., $\mathrm{E}\left[\boldsymbol{u}_{L^{*}}(t)\right]$, differs from the average thermal recovery of the outside tissue computed across the locations $N^{*}$, i.e., $\mathrm{E}\left[\boldsymbol{u}_{N^{*}}(t)\right]$. One way to quantify such a difference is by means of the norm of the difference $\mathrm{E}\left[\boldsymbol{u}_{L^{*}}(t)\right]-\mathrm{E}\left[\boldsymbol{u}_{N^{*}}(t)\right]$, normalized by the total number of frames of the IR sequence in order to compensate for small differences in the acquisition time between patients. The mathematical expression for such a quantification approach for a patient is as follows,

$$
d=\frac{\left\|E\left[u_{L^{*}}(t)\right]-E\left[u_{N^{*}}(t)\right]\right\|}{K-1}
$$

where $E$ stands for the mathematical expectation and $\mathrm{K}$ is the third dimension in the dataset $\boldsymbol{u}$, while $\|$. represents the Euclidean norm of the argument. The farther apart the curves are, the bigger the parameter $d$ and the more likely that the lesion is malignant. We declare the lesion of the patient $l$ as malignant if $d_{l}$ $>\tau$, where $\tau$ is a specified threshold. 


\section{Results}

A cohort study of 102 subjects was performed to investigate the proposed approach. Fifty-eight percent of the subjects were male and from the biopsy results, out of the 102 subjects 59 had benign lesions, 29 had basal-cell carcinoma (BCC), 8 had squamous-cell carcinoma (SCC) and 6 had malignant melanoma (MM).

\subsection{Distribution of the quantification factor}

The distribution of the $d$ parameter for malignant (MM, SCC and BCC) and benign lesions is depicted as a box-and-whisker plot in Fig. 3. Here, the box represents the first and third quartile, the red line is the median of the parameter and the blue dot is the statistical mean. The bars represent the 5\% and $95 \%$ percentiles of the parameter.

It can be seen in Fig. 3 that to classify the lesions with the d parameter, the value of the threshold should be placed between 0.22 and 0.3 because within that range the $25 \%$ percentile of the parameter value for malignant lesions is separated from the $75 \%$ percentile of the parameter for benign lesions. Next, we discuss our approach to select the value of the threshold, $\tau$.

\subsection{Optimal classifier operation}

For our purpose, the value of the threshold, $\tau$, is selected such that the sensitivity (also known as detection probability) and specificity (also known as the complement of the false alarm probability) are jointly maximized.

In order to characterize the relationship between the threshold and the sensitivity and specificity of the algorithm, quantities that define the performance of the algorithm, the empirical receiver-operating characteristic (ROC) curve of the algorithm must be obtained [30]. This is made by continuously changing $\tau$ within the range $\left[\min _{l}\left(d_{l}\right), \max _{l}\left(d_{l}\right)\right]$ and computing the empirical sensitivity and specificity for each value of $\tau$. The plot of the sensitivity vs. the false alarm rate (defined as 1 - specificity) as the threshold is changed generates the empirical ROC curve, which for our study is shown in Fig. 4. 
This curve summarizes the actual performance achieved by the algorithm for different values of the threshold value. For example if one requires the algorithm to detect all of the malignant lesions with indifference to the detection of the benign lesions, then one selects the point where the sensitivity is $100 \%$, for which the specificity is about $50 \%$. If, for example, one requires detection of only the benign lesions, then the selected point ( $0 \%$ false alarm rate) achieves almost $40 \%$ of sensitivity. Therefore, in Fig. 4 the reader can easily see how well (or bad) this algorithm will perform for this particular application.

As was mentioned previously, we require the algorithm to jointly maximize the sensitivity and the specificity. The green circle represents such a maximum, which is achieved for the threshold value $\tau=0.238$. For such a threshold value, the achieved sensitivity is $95.35 \%$ with a $95 \%$ confidence interval (CI) of [88.17\% 100\%]; this implies that under the assumption that this study is representative of the natural occurrences of cancerous lesions, one can correctly classify more than $95 \%$ of the malignant cases. The achieved specificity is $83.05 \%$ with a $95 \%$ CI of [ $73.01 \% 91.87 \%$ ], implying that under the same condition, more than $83 \%$ of the benign lesions will be correctly discharged from the clinic, without requiring a biopsy.

Another performance metric, known as the area under the curve (AUC) computes the area under the ROC curve to measure how close the algorithm under test departs from the perfect classifier (a perfect classifier has an AUC of 100\%) [30]; for our clinical study the achieved AUC is $95.03 \%$ with a $95 \%$ CI [ $90.31 \%$ $99.76 \%]$

\section{Discussion}

To the best of our knowledge, this is the first study of DTI that comprises a large amount of patient data (>100 lesions) and yields excellent performance in the classification of malignant lesions, achieving an AUC of 95\%. Even though further development and clinical validation are necessary, the results are promising and suggest that the DTI approach working jointly with the proposed analysis algorithm has 
the potential to provide a fast, accurate, non-contact, and non-invasive way to screen for common types of skin cancer. As such, it has the potential to significantly reduce the number of biopsies performed on suspicious lesions due to its high specificity while its high sensitivity ensures that cancerous lesions are biopsied.

The principal strength of our study was the introduction of a standard methodology for the subject data analysis. The classification algorithm only requires the selection of the lesion area on the visible image, which is a common requirement for diagnostic tools. We are aware that our study may represent an upper bound of the actual performance of DTI for skin cancer detection and, therefore, more data is required to either validate or reject our approach. Moreover, the quality of the data depends on the subject's ability to maintain a steady and comfortable position for two minutes. This makes the lesions in the head region difficult to image and analyze. In addition, the presence of hair (a natural thermal diffusor) adversely affects the data processing and analysis.

Although we defined the $d$ parameter that directly accounts for differences in the thermal recovery and indirectly accounts for imperfections in the cooling of the skin, we are not certain of the reasons that lead such a parameter to perform as a good classifier. Other groups have performed extensive surveys [31] that may explain that such a difference is due to thermal diffusion, blood perfusion or even the different metabolic heat generated by the cancerous cells. We have started studies to understand the physiology of the lesion.

\section{Conclusions}

We performed a cross-sectional study of 102 subjects using DTI to classify the malignancy of lesions. To the best of our knowledge, this is the first time that a fast and non-invasive screening approach based on DTI achieves these levels of performance.

The obtained results demonstrate that DTI with a properly standardized methodology is a promising tool for detecting skin cancer. Moreover, it seems that the selection of pixels from normal skin and the suspect 
lesion with the same initial temperature is the key enabling-step for the utilization of DTI for skin cancer detection.

\section{Acknowledgements}

Skinfrared, LLC internal Research \& Development funds and the NSF Phase-I IIP-1315372 grant supported this work. Support from the UNM Dermatology Clinic staff is also acknowledged. S. E. Godoy is supported by the Comisión Nacional de Investigación Científica y Tecnológica (CONICYT), Chile, The New Mexico Cancer Nanotechnology Training Center (CNTC), University of New Mexico and the University of Concepción, Chile.

\section{References}

[1] Cancer Facts \& Figures 2014, Atlanta: American Cancer Society, 2014. Available Online; Last Accessed: July 2014.

[2] H. W. Rogers, M. A. Weinstock, A. R. Harris, M. R. Hinckley, S. R. Feldman, A. B. Fleischer, B. M. Coldiron, Incidence estimate of nonmelanoma skin cancer in the United States, 2006, Archives of Dermatology 146 (2010) 283-287.

[3] N. R. Abbasi, H. M. Shaw, D. S. Rigel, R. J. Friedman, W.H. McCarthy, I. Osman, A. W. Kopf and

D. Polsky, "Early diagnosis of cutaneous melanoma: revisiting the ABCD criteria," JAMA 292 (22): 2771-2776, 2004

[4] L. Thomas, P. Tranchand, F. Berard, T. Secchi, C. Colin, G. Moulin, "Semiological Value of ABCDE Criteria in the Diagnosis of Cutaneous Pigmented Tumors,” Dermatology 197 (1): 11-17, 1998

[5] C. Benellii, E. Roscetti and V. Dal Pozzo, “The dermoscopic (7FFM) versus the clinical (ABCDE) diagnosis of small diameter melanoma,” Eur J Dermatol. 10 (4): 282-287, 2000

[6] H. G. Welch, S. Woloshin and L. M. Schwartz, "Skin biopsy rates and incidence of melanoma: population based ecological study," BMJ 2005; 331:481 
[7] V. Ahlgrimm-Siess, M. Laimer, E. Arzberger, R. Hofmann-Wellenhof, "New diagnostics for melanoma detection: from artificial intelligence to RNA microarrays," Future Oncol. 8 (7) : 819-827, July 2012

[8] A. Kardynal and M. Olszewska, "Modern non-invasive diagnostic techniques in the detection of early cutaneous melanoma," J Dermatol Case Rep. 8(1): 1-8, Mar 31, 2014

[9] M. Carrara, A. Bono, C. Bartoli, A. Colombo, M. Lualdi, D. Moglia, N. Santoro, E. Tolomio, S. Tomatis, G. Tragni, M. Santinami, R. Marchesini, "Multispectral imaging and artificial neural network: mimicking the management decision of the clinician facing pigmented skin lesions," Phys Med Biol. 52 (9): 2599-2613, 2007

[10] D. S. Rigel, M. Roy, J. Yoo, C. J. Cockerell, J. K. Robinson and R. White "Impact of Guidance From a Computer-Aided Multispectral Digital Skin Lesion Analysis Device on Decision to Biopsy Lesions Clinically Suggestive of Melanoma,” Arch Dermatol. 148 (4): 541 543,2012

[11] I. Quinzán, J. M. Sotoca, P. Latorre-Carmona, F. Pla, P. García-Sevilla and E. Boldó, “Band selection in spectral imaging for non-invasive melanoma diagnosis," Biomed Opt Express 4 (4): $514-519,2013$

[12] I. Tromme, L. Sacré, F. Hammouch, C. Legrand, L. Marot, P. Vereecken, I. Theate, P. van Eeckhout, P. Richez, J. F. Baurain, L. Thomas, N. Speybroeck, “Availability of digital dermoscopy in daily practice dramatically reduces the number of excised melanocytic lesions: results from an observational study,” Br J Dermatol.167 (4), pp. 778-786, 2012 
[13] A. Saez, C. Serrano and B. Acha, "Model-Based Classification Methods of Global Patterns in Dermoscopic Images," IEEE Transactions on Medical Imaging, vol.33, no.5, pp. 1137-1147, 2014

[14] G. Pellacani, B. De Pace, C. Reggiani, A. M. Cesinaro, G. Argenziano, I. Zalaudek, H. P. Soyer and C. Longo, "Distinct melanoma types based on reflectance confocal microscopy," Experimental Dermatology, Exp Dermatol. 23(6), pp.414-418, June 2014

[15] A. Dancey, B. Mahon, S. Rayatt, A review of diagnostic imaging in melanoma, Journal of Plastic, Reconstructive \& Aesthetic Surgery 61, pp. 1275-1283, 2008

[16] R. Kleinerman, T. B. Whang, R. L. Bard, E. S. Marmur, "Ultrasound in dermatology: Principles and applications," Journal of the American Academy of Dermatology, Volume 67, Issue 3, pp 478-487, September 2012

[17] R. E. Hunger, R. Della Torre, A. Serov and T. Hunziker, "Assessment of melanocytic skin lesions with a high-definition laser Doppler imaging system," Skin Research and Technology 18 (2), pp. 207-211, 2012

[18] Z. Hamdoon, W. Jerjes, T. Upile and C. Hopper, “Optical coherence tomography-guided photodynamic therapy for skin cancer: Case study," Photodiagnosis and Photodynamic Therapy 8, pp. 49-52, 2011

[19] L. Themstrup, C.A. Banzhaf, M. Mogensen, G.B.E. Jemec, "Optical coherence tomography imaging of non-melanoma skin cancer undergoing photodynamic therapy reveals subclinical residual lesions," Photodiagnosis and Photodynamic Therapy 11 (1), pp. 7-12, March 2014 
[20] A. R. Cukras, "On the Comparison of Diagnosis and Management of Melanoma Between Dermatologists and MelaFind.” JAMA Dermatol. 2013; 149(5):622-623

[21] N. Nandanan, J. Fisher, D. M. Siegel and O. Markowitz in "Optical Coherence Tomography Imaging of Onychomycosis," Presented at EADV, Florence, 2012; available online http://www.vivosight.com/clinical-presentations/ Last Accessed: May 2014.

[22] B. B. Lahiri, S. Bagavathiappan, T. Jayakuman, J. Phillip, Medical applications of infrared thermography: A review, Infrared Physics \& Technology 55 (2012) 221-235.

[23] M. Anbar, Quantitative Dynamic Telethermometry in Medical Diagnosis and Management, CRC Press, Boca Raton, FL, 1994.

[24] T. Buzug, S. Schumann, L. Pfamann, U. Reinhold, J. Ruhlmann, "Functional infrared imaging for skin-cancer screening," in: Engineering in Medicine and Biology Society, 2006. EMBS '06, 28th Annual International Conference of the IEEE, pp. 2766-2769

[25] M. P. Cetingul and C. Herman, Quantification of the thermal signature of a melanoma lesion, Int. Journal of Thermal Science 50 (2011) 421-431

[26] C. Harris and M. Stephens, "A combined corner and edge detector," Proceedings of the 4th Alvey Vision Conference, pp. 147-151, 1988

[27] A. Ardeshir Goshtasby, "2-D and 3-D Image Registration: for Medical, Remote Sensing, and Industrial Applications,” Wiley Press, 2005

[28] M. A. Fauci, R. Breiter W. Cabanski, W. Fick, R. Koch, J. Ziegler and S. D. Gunapala, Medical infrared imaging - differentiating facts from fiction and the impact of high precision quantum well infrared photodetector camera systems, and other factors, in its reemergence, Infrared Physics \& Technology 42 (2001) 337-344. 
[29] Hancock, Matthew. 18.303 Linear Partial Differential Equations, Fall 2006. (MIT OpenCourseWare: Massachusetts Institute of Technology), http://ocw.mit.edu/courses/mathematics/18-303-linear-partialdifferential-equations-fall-2006 (Accessed 31 Jul, 2014). License: Creative Commons BY-NC-SA

[30] Tom Fawcett. ROC graphs: Notes and practical considerations for data mining researchers. Technical Report HPL-2003-4, HP Laboratories, Palo Alto, CA, January 2003.

[31] M. P. Cetingul and C. Herman, "A heat transfer model of skin tissue for the detection of lesions: sensitivity analysis,” Phys. Med. Biol. 55 5933, 2010

\section{Figure captions}

Fig. 1: Example of one square plastic marker used in the data acquisition step; the biggest rectangle that can be drawn within the opening of the marker is defined by the points $\left(i_{\min } j_{\min }\right)$ and $\left(i_{\max } j_{\max }\right)$, labeled in the inset. The suspicious lesion is also labeled and surrounded by the ellipse (light blue) drawn by the user

Fig. 2: Dual exponential model fitting approach followed in this work to minimize the influence of noise when compensating for the non-uniform initial temperature distribution. Two representative thermal recoveries are used $(\boldsymbol{u}(1,1)$ and $\boldsymbol{u}(100,100)$, specifically) to show the model fitting.

Fig. 3: Distribution of the quantification factor, $d$, for the cohort sample used in this study. It can be noted that the $25 \%$ and $75 \%$ percentiles of the benign cases (59 subjects) and malignant cases (43 subjects), respectively, are sufficiently apart to permit good classification results

Fig. 4: Empirical receiver operating characteristic (ROC) curve obtained for the cohort sample used in this study (solid red line) and the random guess result (blue dashed line). The maximum sensitivity and specificity as well as the area under the curve (AUC) are given as insets. The 95\% confidence intervals for all the performance metrics are given in squared parenthesis. 


\section{Figures}

Figure 1:

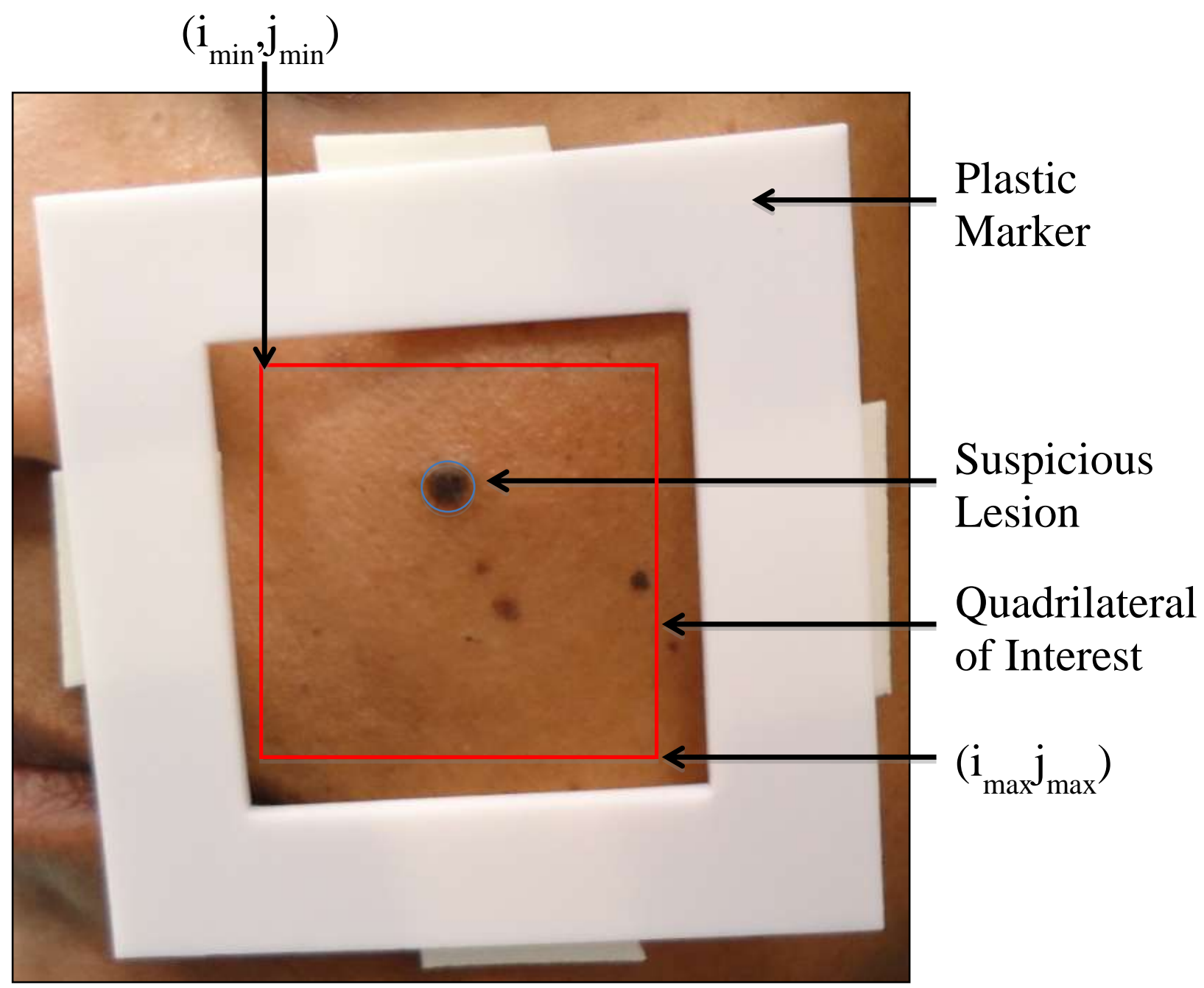


Figure 2:

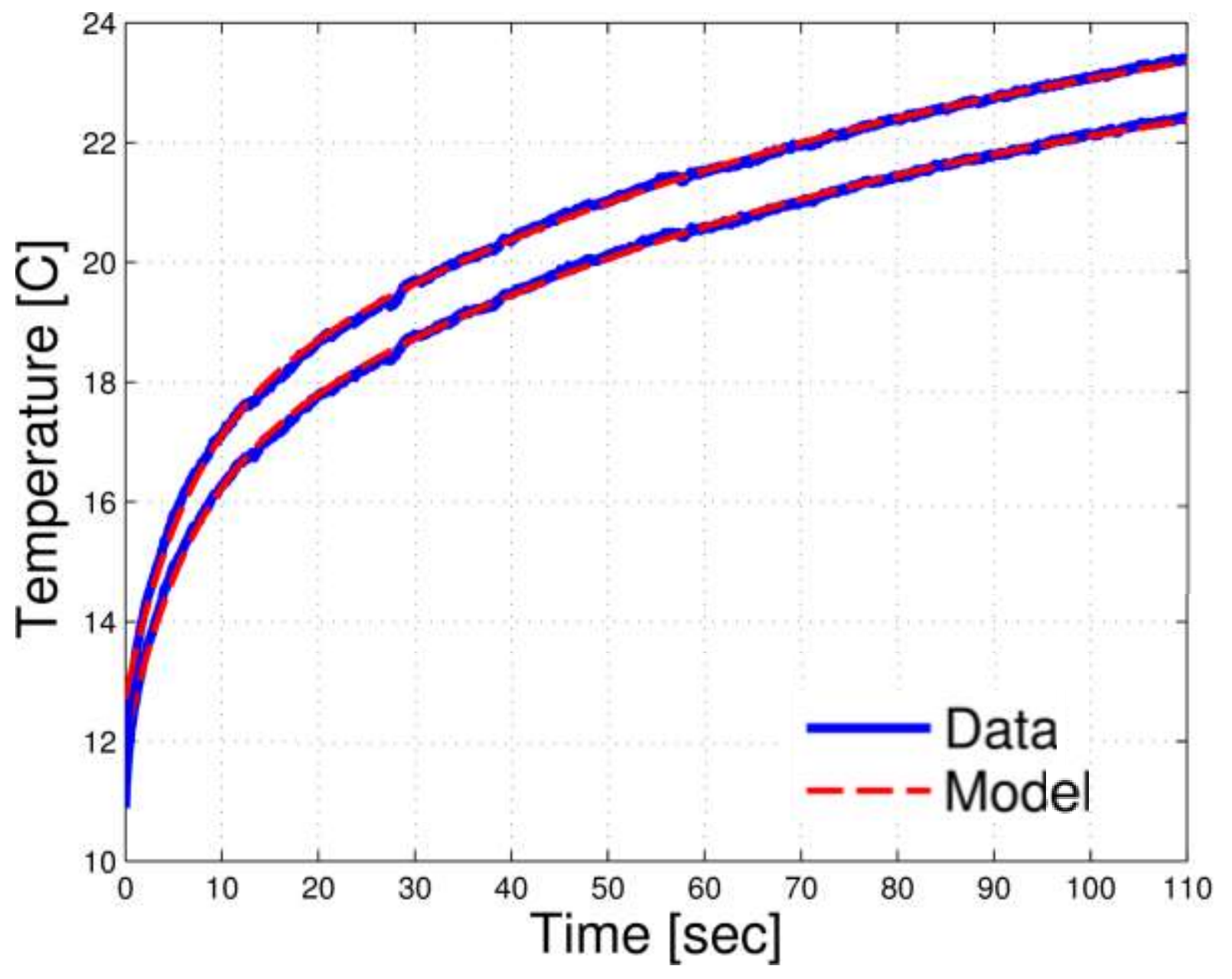


Figure 3:

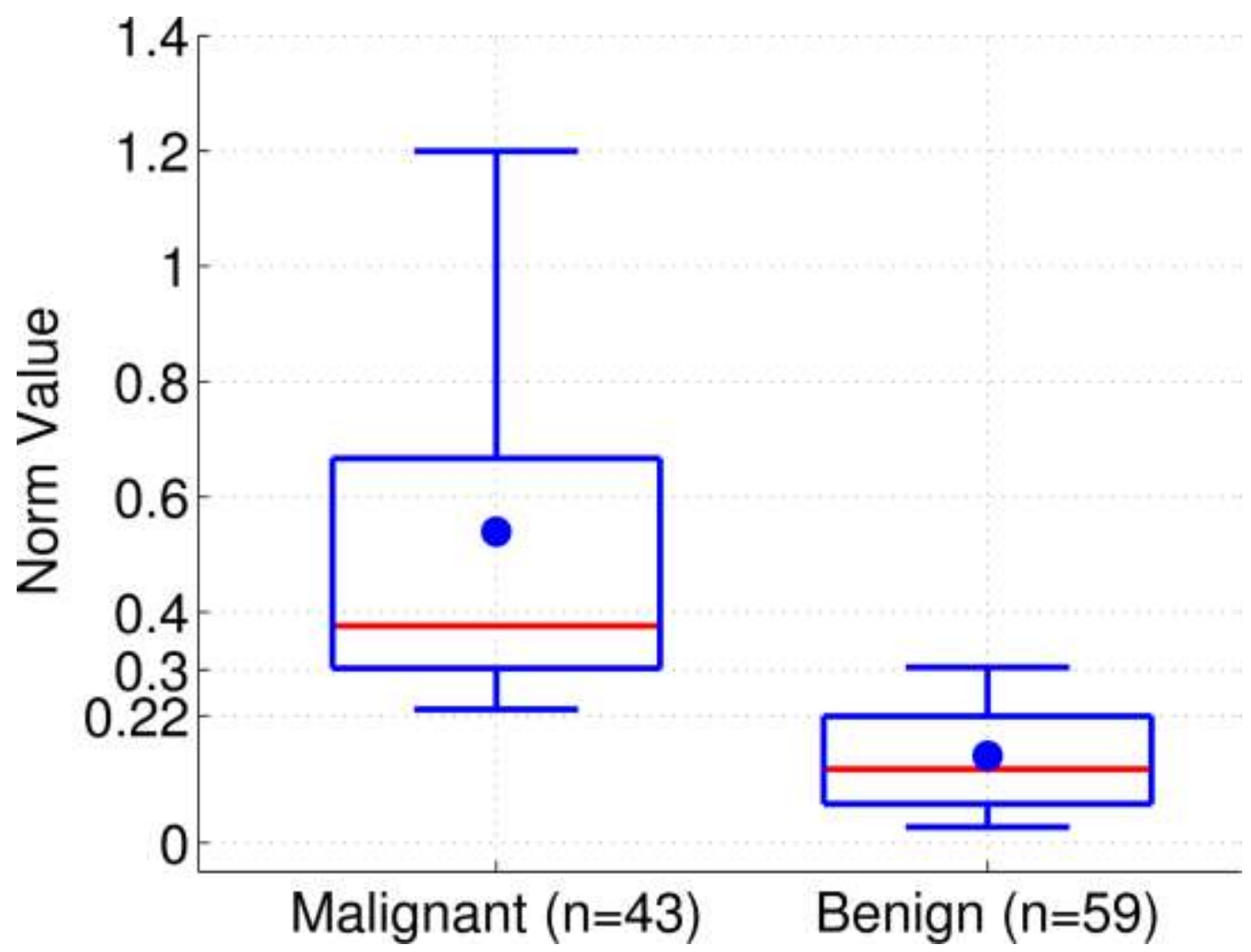


Figure 4:

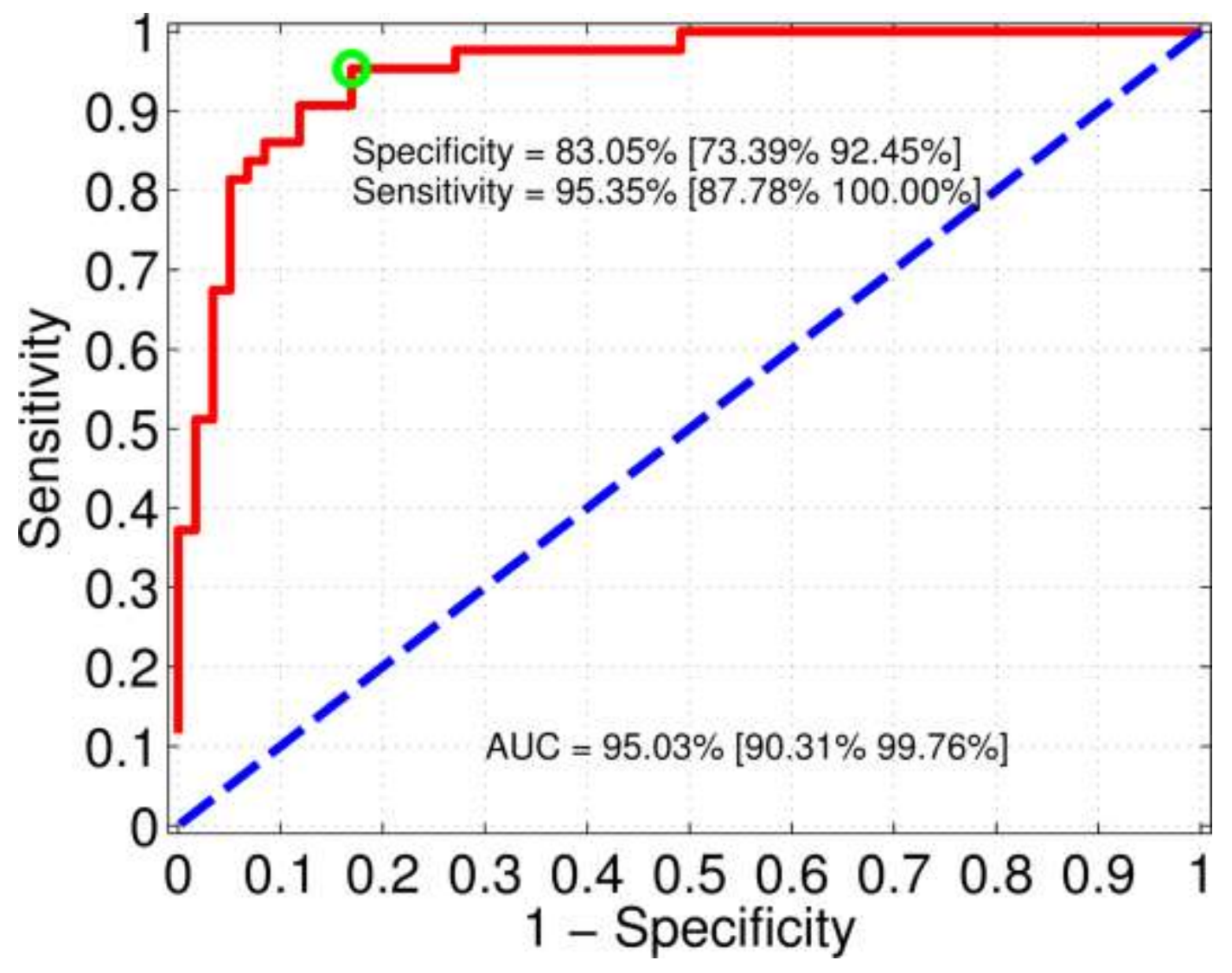

